\title{
COVID-19 and the Elderly: Why Does the Immune System Matter?
}

\author{
Chalise $\mathrm{HN}^{1,2 *}$ and Rosenberg $\mathrm{E}^{3}$ \\ ${ }^{1}$ Department of Public Health, Pokhara University, Nepal \\ ${ }^{2}$ Population Association of Nepal, Nepal \\ ${ }^{3}$ Department of Sociology, Appalachian State University, USA
}

*Corresponding author: Dr. Hom Nath Chalise, Faculty Member, Department of Public Health, Nobel College affiliated to Pokhara University, Sinamangal, Kathmandu, Nepal, Tel: +977 9851012622; Email: chalisehkpp@gmail.com

\section{Abstract}

The global population is rapidly aging. People are living longer due to advances in education, technology, medicine, food distribution, and public health. While the COVID-19 pandemic has significant global impacts, in many countries the elderly face threats and challenges that are unique and disproportionately severe. One such threat is that aging results in a decline in immune function, meaning elderly bodies respond more slowly and less effectively to external threats like COVID-19. Responses at individual, family, community and societal levels should take into account the heightened vulnerability of older adults during this pandemic.

Keywords: Coronavirus; COVID-19; Older adults; Immune system

Abbreviations: COVID-19: Coronavirus Disease; WHO: World Health Organization.

\section{Introduction}

The coronavirus widely accepted as having originated in and spread from Wuhan, China, is considered by the World Health Organization (WHO) to be a severe acute respiratory syndrome coronavirus 2, or SARS-CoV-2 [1]. The resultant coronavirus disease is popularly called COVID-19. Until February 2020, few nations outside China reported COVID-19 cases. In March 2020, based on confirmed cases, the virus began spreading rapidly: initially in South Korea, by the second week of March in Italy, Spain and other European countries, and in the third week of March to the United States [2]. On 11 March WHO declared COVID-19 a pandemic [3]. As of 27 June there are more than 10 million confirmed cases, with more than 500,000 deaths and less than 5.5 million recovered [4]. The latest data also show that COVID-19 cases rates are increasing more rapidly in poor and developing countries than in wealthier and more developed nations $[4,5]$.
Rapid population aging is a global phenomenon, regardless of a nation's level of development [6]. Today, people are living longer than ever before due to advances in education, technology, medicine, food distribution, and public health [7]. An aging population impacts almost every area of government policy and presents a number of socioeconomic and health challenges. The main objective of this paper is to explore older adults' increased vulnerability to COVID-19 in terms of the function of the immune system, and to suggest that local, regional and national responses consider the higher risk of COVID-19 to older adults.

\section{COVID-19 and the Elderly}

Around the world, people are suffering from COVID-19. It is, after all, a pandemic. But the virus death rates are unequal across age groups. Since the COVID-19 death rate increases sharply with age, the larger the older population, the greater the challenges for communities and societies. And within older populations, the older subgroups (e.g., age $80+$ ), which are most vulnerable to infection and physiologically least able to recover, are growing more rapidly than younger 
subgroups (e.g., 65-74). Thus cross-national differences in population age compositions partly explain the spread and the crude fatality measures of the disease. Considering population age structure is therefore critically important in assessing, for example, the potential impact of COVID-19 on mortality in Europe -- especially southern Europe, the world's oldest region [8]. Data shows 29 of the 30 nations with the highest percent of older people are in Europe [9].

Although people of all ages are affected by COVID-19, older adults are at disproportionate risk of contracting and dying from the virus. WHO data from April 2020 shows more than 95\% of COVID-19 deaths were among people over 60 years of age, and more than half of all deaths occurred in people age 80+ [8]. In March, the Chinese Centers for Disease Control and Prevention offered data showing COVID-19 mortality increased with age: the fatality rate was $3.6 \%$ for adults in their $60 \mathrm{~s}, 8 \%$ for those in their 70 s, and $14.8 \%$ for people age $80+[10]$. A report from Sweden showed that $90 \%$ of COVID-19 deaths were among people age 70+ [11]. And as of mid-May in New York City, nearly three-quarters (73.6\%) of COVID-19 deaths were people age 65+. Nearly half (48.7\%) of New York's COVID-19 deaths were people age 75+ [12].

Nursing home populations are at high risk of coronavirus infection and death. For example, 53\% of Belgium's COVID-19 deaths occurred in care homes. In Canada the figure is $62 \%$, in France between 39.2-51\%, and in Spain 67\% [11]. Many studies show older adults are more likely than younger people to have pre-existing conditions such as cardiovascular disease, diabetes, chronic respiratory disease, hypertension, and cancer. Further, the inevitable natural deterioration of the immune system makes it harder for older adults to fight off infection. Obesity and smoking also weaken the immune system and are thus associated with increased risk. There are gender correlations as well: in Italy, higher risk has been reported in men than in women [13], perhaps due to their higher smoking rates and subsequent comorbidities.

But age is not a completely determining risk factor for severe disease. In some places or situations, younger people are also at very high risk during a pandemic. A recent Nepal study found that people below age 50 account for more than 80 percent of COVID-19-infected persons and a similarly disproportionate share of COVID-19 deaths [14]. And anecdotal evidence suggests old age per se is not a COVID-19 death sentence: it has been reported that even centenarians who were admitted to hospital for COVID-19 have made complete recoveries. Among them are a 113-year-old Spanish woman, a 107-year-old Turkish woman, a 106-yearold British woman, a 104-year-old Korean and a 102-yearold woman from Singapore.

\section{Aging and the Immune System}

According to Sadighi Akha AA, aging is a multifaceted process, involving numerous molecular and cellular mechanisms in the context of different organ systems. An important part of aging is a set of functional and structural alterations that weaken the immune system. This can be manifested as a decreased ability to fight infection, diminished response to vaccination, increased incidence of cancer, higher prevalence of autoimmunity, and constitutive low-grade inflammation, among others [15]. The immune system thus has a vital role in health; in general, however, older adults have a weaker immune system and consequently are less resilient in fighting off infection. The immune system protects the body from outside invaders such as bacteria, viruses, fungi, and toxins. According to John Hopkins Medicine [16], the immune system, made up of different organs, cells, and proteins, works to protect the body against disease. This protection is called immunity. The immune system has main two parts. The innate (natural) immune system, which one is born with, provides general protection. The adaptive immune system is what the body develops when exposed to microbes or chemicals released by microbes-that is, outside invaders. These two immune system dimensions work together, discriminating between self and foreign components and reacting against any foreign molecule different from the body's own structure.

According to Márquez EJ, et al. [17], our immune system has two sets of defenses against viruses and other pathogens: a first-line army of cells, called leukocytes that attack invading microbes within minutes to hours, and a second-line force of precisely targeted antibodies and $\mathrm{T}$ cells that engage the invading microbes up to several days later. $\mathrm{T}$ cells produce virus-fighting chemicals. As the body ages, it has fewer $\mathrm{T}$ cells. By puberty, the thymus is producing tenfold fewer $\mathrm{T}$ cells than it did in childhood; by age 40 or 50 , there is another tenfold drop. That leaves the body severely depleted of $\mathrm{T}$ cells that have not yet been programmed to defend against a specific microbe. Fewer such "naïve T cells" means fewer able to be deployed against a never-before-seen microbe [17]. It is clear, then, why and how immunity weakens with age, and why older people are less likely to be able to react effectively to microorganisms their bodies haven't encountered before. Still, it is not chronological age alone that determines how one does in the face of a life-threatening infection such as COVID-19. Pre-existing conditions, such as chronic diseases, and frailty can be as or more important than chronological age [18].

There is ongoing research on maintaining a strong immune system, retarding immune system decline, and strengthening weakened immune systems. But to date no 
definitive solution has emerged. We know why immunity decreases with age, but not how to prevent this. Put simply, you have fewer immune cells as you age, and the ones you do have don't communicate with each other as well. Thus they take longer to react to harmful germs and you are more likely to get sick. In addition, the aging body produces fewer immune cells, including white blood cells, which can slow recovery from injuries, infection, and illness [19].

In general, then, aging results in a decline in immune function. Older adults are not necessarily immune deficient, and there is great individual variation. The elderly can, but usually do not, respond effectively to COVID-19. Thus public health efforts, which of course include sheltering in place, social distancing, and masks, should also focus on immune system health promotion for older people. Activities that are correlated with immune system maintenance or even boosting (thus increasing resistance to various viruses) include getting a flu vaccination, eating a healthy diet, being physically active, lowering one's stress level, getting adequate sleep, maintaining a healthy weight, avoiding tobacco, and spending time outdoors.

Finally, COVID-19 is most likely to be lethal to older adults with pre-existing health conditions. Maintaining good health through health promotion activities and a healthy lifestyle play crucial roles in minimizing immune system decline and maximizing disease resistance. People who age healthily are less at risk. Individuals, families, societies, governments, policymakers, and international development institutions must invest in culturally appropriate programs and services that promote active aging. This will, simultaneously, maintain immune system strength and overall good health.

\section{References}

1. Chalise HN (2020) COVID-19 situation and challenges of Nepal. Asia Pac J Public Health.

2. Chalise HN (2020) COVID-19 situation in Nepal. Arch Psychiatr Ment Health 4: 33-34.

3. WHO (2020) WHO announces COVID-19 outbreak a pandemic. World Health Organization, Geneva, Switzerland.

4. Worldometer (2020) COVID-19 coronavirus pandemic, USA.
5. Chalise HN (2020) South Asia is more vulnerable to COVID-19 pandemic. Arch Psychiatr Ment Health 4: 4647.

6. Chalise HN (2019) Aging: Basic Concept. Am J Biomed Sci \& Res 1(1): 8-10.

7. Chalise HN, Rosenberg E (2019) Social and Health Status of Community-Dwelling Older Adults in Nepal. Advances in Aging Research 8(4): 63-74.

8. WHO (2020) Statement-Older people are at highest risk from COVID-19, but all must act to prevent community spread. World Health Organization.

9. Population Reference Bureau (2020) Countries with the oldest populations in the world.

10. Health \& Pharmaceuticals (2020) Development of novel coronavirus COVID-19 case fatality rate (CFR) in China from January 19 to June 25, 2020.

11. Medical News Today (2020) The impact of the COVID-19 pandemic on older adults.

12. Worldometer (2020) Age, Sex, Existing Conditions of COVID-19 Cases and Deaths, USA.

13. Livingston E, Bucher K (2020) Coronavirus Disease 2019 (COVID-19) in Italy. JAMA.

14. Chalise HN, Dhungana HN (2020) Fears of COVID-19 catastrophe as Nepal reports death from new Coronavirus. J Phys Med Rehabil Disabil 6: 047.

15. Sadighi Akha AA (2018) Aging and the immune system: An overview. J Immunol Methods 463: 21-26.

16. Johns Hopkins Medicine (2020) The immune system.

17. Márquez EJ, Chung C, Marches R, Rossi RJ, Nehar-Belaid $D$, et al. (2020) Sexual-dimorphism in human immune system aging. Nat Commun 11: 751.

18. STAT (2020) What explains Covid-19's lethality for the elderly? Scientists look to 'twilight' of the immune system.

19. Montecino-Rodriguez E, Berent-Maoz B, Dorshkind K (2013) Causes, consequences, and reversal of immune system aging. The Journal of clinical investigation 123(3): 958-965. 